\title{
A reciprocating pin-on-plate test-rig for studying friction materials for holding brakes
}

\author{
Poulios, Konstantinos; Drago, Nicola; Klit, Peder; De Chiffre, Leonardo
}

Published in:

Wear

Link to article, DOI:

10.1016/j.wear.2013.12.023

Publication date:

2014

Document Version

Peer reviewed version

Link back to DTU Orbit

Citation (APA):

Poulios, K., Drago, N., Klit, P., \& De Chiffre, L. (2014). A reciprocating pin-on-plate test-rig for studying friction materials for holding brakes. Wear, 311(1-2), 40-46. https://doi.org/10.1016/j.wear.2013.12.023

\section{General rights}

Copyright and moral rights for the publications made accessible in the public portal are retained by the authors and/or other copyright owners and it is a condition of accessing publications that users recognise and abide by the legal requirements associated with these rights.

- Users may download and print one copy of any publication from the public portal for the purpose of private study or research.

- You may not further distribute the material or use it for any profit-making activity or commercial gain

- You may freely distribute the URL identifying the publication in the public portal 


\title{
A reciprocating pin-on-plate test-rig for studying friction materials for holding brakes
}

\author{
K. Poulios ${ }^{a, *}$, N. Drago ${ }^{b}$, P. Klit ${ }^{a}$, L. De Chiffre $^{b}$ \\ ${ }^{a}$ Technical University of Denmark, Department of Mechanical Engineering, Solid Mechanics Nils \\ Koppels Allé , Building 404, 2800 Kgs. Lyngby \\ ${ }^{b}$ Technical University of Denmark, Department of Mechanical Engineering, Manufacturing \\ Engineering Produktionstorvet, Building 425, 2800 Kgs. Lyngby
}

\begin{abstract}
This paper refers to testing of friction materials for holding brakes. In contrast to the more typical case of high energy brakes, holding brakes operate usually in a reciprocating sense, at very low sliding speeds and allow significantly higher clamping pressures. The design of a reciprocating pin-on-plate test-rig for studying the evolution of wear by monitoring the pin height reduction using Eddy-current proximity sensors, is presented. Moreover, a new mechanism for recording the friction force is suggested. Apart from the design of the test-rig, friction force and wear rate measurements for two different friction materials running against an unhardened steel surface are presented as a usage case.
\end{abstract}

Keywords: pin-on-plate, wear, friction, holding brakes

\section{Introduction}

Holding brake applications are generally not as extensively studied as typical high energy brake applications. Their particularities compared to high energy brakes include low sliding speeds, high clamping pressures, bidirectional motion and low temperatures. Theoretically, holding brake materials should experience relative sliding only in completely released state and thus exhibit insignificant wear. In praxis however, it is not uncommon that during sliding, significant normal load is present, e.g. either because the brake is not supposed to be released completely or because it is designed to be functioning as sliding bearing at the same time. Typical holding brake applications are e.g. the yaw system brakes in cranes and wind turbines.

The clamping force in holding brakes can be either passive, generated through preloaded springs or active, generated through hydraulic or pneumatic actuators. Especially in the first case, the wear of the friction materials is a very important parameter to take into account for a proper operation of the equipment in long term. But wear is also important for active holding brakes, in cases where very long lifetime and maintenance intervals are required.

* Corresponding author

Email address: kopo@mek.dtu.dk (K. Poulios) 
Friction material candidates for holding brake applications include paper like or woven fiber composites but also thermoplastics either in pure form or reinforced with short fibers, e.g. glass, aramid and carbon fibers. Available friction and wear-rate data for such friction materials usually refer to relatively low pressure ranges and relatively high sliding velocities, typical for sliding bearing applications, see e.g. 1]. Regarding holding brakes, a sliding speed range between 1 and $50 \mathrm{~mm} / \mathrm{sec}$ can be considered as indicative, while the clamping pressure can vary from $2 \mathrm{MPa}$ to more than $20 \mathrm{MPa}$.

Such operational conditions can be reproduced in both pin-on-disc and pin-on-plate test-rigs, see Sec. 9.2.8 and 9.2.11 of [2]. Pin-on-plate test-rigs are inherently reciprocating while pin-on-disc test-rigs can be set up to operate also in reciprocating sense, like in $[3]$.

In literature, different methods for quantifying wear in pin-on-disc and pin-on-plate testing are suggested. ASTM standards [4] and [5] as well as Appendix B, Sec. 3 of [6], provide a more detailed discussion on the different possibilities for wear measurements during and after the testing. After the test, measuring weight loss and dimensional changes can provide information about pin wear, while profilometric measurements can indicate wear on the disc or plate. During the test, the so called wear displacement, i.e. the displacement of the pin with respect to the counter surface, can be monitored. For this purpose, the above mentioned ASTM standards suggest using a linear displacement sensor that records the vertical displacement of the loading arm with respect to the testrig frame. The present paper suggests a slightly improved implementation of monitoring the wear displacement utilizing Eddy-current proximity sensors [7, mounted very close to the pin sample.

An important aspect in both pin-on-disc and pin-on-plate testing is the form of the pin end. Usually pins with spherical or tapered ending are recommended, like e.g. in [4] and [5, but other forms including flat ended pins are also possible and discussed in more detail in Sec. 9.2.8 of [2]. The main advantage of using spherical pins is that a very high contact pressure can be achieved through a moderate normal force. However, the contact pressure is not even approximately constant and its actual distribution depends on the wear process. Moreover, as wear progresses the contact area grows resulting to a decreasing average pressure. Flat ended pins do not exhibit these drawbacks, however they may suffer from high edge loads when the pin material is stiffer than the counter surface material or when material pairs of similar stiffness are tested. Edge loads do not represent an issue for testing typical friction materials for holding brakes against a steel surface because these materials are normally considerably more compliant than steel.

With respect to the measurement of the friction force, there are basically two possibilities. One is to record the reaction forces on the pin side and the second option is to record the reaction forces or moments on the plate or disc side, Sec. 4.2 of 8 . Utilizing strain gauges or commercial force transducers for measuring these forces is a very common approach, Sec. 8.6 of [2] and Appendix B, Sec. 3 of [6]. The present paper suggests a new mechanism for recording the reaction of the friction force on the loading arm, the pin is mounted on, utilizing common tensile/compressive force transducers (load-cells).

The normal force applied to the pin can be exerted either by hydraulic actuators or a dead weight and possibly through a lever arm, Appendix B, Sec. 1 of [6]. The normal force can also be monitored through a force transducer or it may be assumed as known if it was appropriately calibrated before the test, Appendix B, Sec. 3 of [6]. 


\section{Methods}

\subsection{The pin-on-plate test-rig design}

Fig. 1 illustrates the concept of the implemented pin-on-plate test-rig. The tested material is in form of a flat ended cylindrical pin (1) that is vertically loaded through a dead weight (3) and a lever arm (2). This loading arm is connected to the intermediate lever (5) at the pivot point (A). With respect to the fixed sub-frame (6), lever (5) is free to rotate around pivot (B). Its rotation is restricted exclusively through load-cell (4). According to the equilibrium of moments for lever (5) around pivot (B), the horizontal force component transferred from loading arm (2) to lever (5) through pivot (A), generates a proportional force in load-cell (4). By choosing the vertical distances between pivot (B) and pivots (A) and (C) to be equal, the horizontal force recorded by load-cell (4) is equal to the horizontal force component applied to pivot (A) and consequently equal to the friction force exerted on pin (1).

The counter surface consists of the interchangeable insert plate (8) which is mounted on the moving plate (7). Plate (7) is connected with the stationary frame (9) through linear bearings and it is driven by a motor through a worm gear and a threaded shaft that are not included in the illustration. Its motion is linear apart from a limited distance near the stroke ends, where the plate motion is reversed.

This test-rig concept allows multiple instances of the loading arm substructure (1-6) and the insert plate (8) to be implemented against a common moving plate (7). The tests presented in the next section were conducted on a quadruplet implementation of the here presented pin-on-plate test-rig.

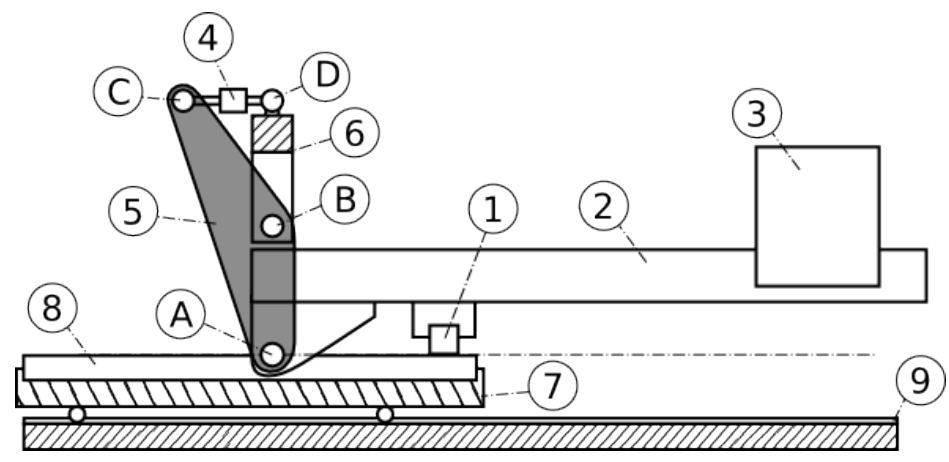

Figure 1: Pin-on-plate test-rig design

The construction of pivots $(\mathrm{A})$ and $(\mathrm{B})$ is a practical aspect that requires some further attention. Friction in pivot (A) affects the normal force applied on the pin, while friction in pivot (B) can yield to a reduced measured force in load-cell (4). In the present work, oil lubricated bronze bushings were utilized for both pivots (A) and (B). In comparison to roller element bearings, lubricated sliding bushings exhibit relatively higher static friction. However, they are more robust and they can consequently be designed with a smaller diameter so that the total friction torque will remain low. Moreover, roller element bearings under stationary load are prone to plastic deformation between the roller elements and the bearing races, which may compromise the function of the bearing and yield to unpredictable behavior. 


\subsection{Wear displacement monitoring}

Fig. 2 shows the structure that supports the pin made of the sample material. Pin (1a) with dimensions of $10 \mathrm{~mm}$ in diameter and $8 \mathrm{~mm}$ in height is glued in holder (1b) which is bolted with the intermediate plate (1c). The purpose of the intermediate plate (1c) is to provide a rigid support for the Eddy-current proximity sensor (11). Plate (1c) is connected directly to loading arm (2) and its position in height is adjustable through an appropriate screw (10).

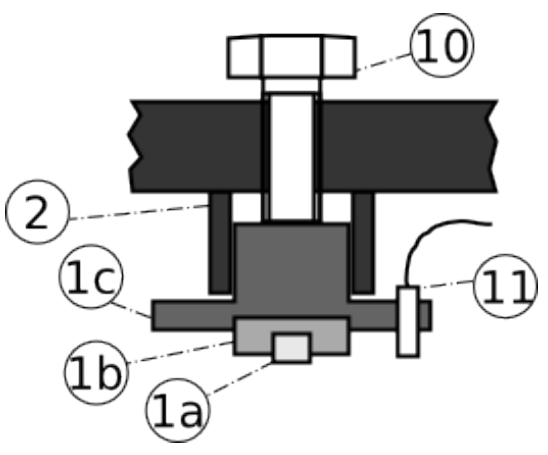

Figure 2: Pin holder design

The Eddy-current proximity sensor (11) provides a signal that is proportional to the gap between its free end and the counter surface (8). In case that the protruding part of sensor (11) with respect to its holder (1c) is too small its linearity may be disturbed significantly, because the steel part (1c) intersects the magnetic field of the sensor. For the $5 \mathrm{~mm}$ in diameter sensor that was used in our implementation, the holding system shown in Fig. 2 was dimensioned in such a way that a minimum distance of $7 \mathrm{~mm}$ between the free end of sensor (11) and its holder (1c) was available, when the sensor was adjusted at a distance of $1.5 \mathrm{~mm}$ from the counter surface (8). The linearity of the assembled sensor was verified by mounting the compound (1b), (1c) and (11) on a height gauge and varying its vertical position in steps of $0.1 \mathrm{~mm}$. At the same time the linearity coefficients between measured signal and monitored gap were specified for a gap range between 0.5 and $2.5 \mathrm{~mm}$.

In order to estimate the wear displacement based on the monitored gap, one has to also take into account the different axial positions of pin (1a) and sensor (11) with respect to the pivot point (A). The wear displacement $\delta h$ is equal to the difference between the monitored gap $g$ at the current time point during a test and the gap $g_{0}$ at a reference time point, multiplied by a geometrical factor:

$$
\delta h=\frac{x_{(1)}}{x_{(11)}} \cdot\left(g-g_{0}\right)
$$

with $x_{(1)}$ and $x_{(11)}$ representing the axial distance from pivot point (A) to the center of the pin and the Eddy-current sensor respectively.

It should be noted that due to geometrical deviations, elastic deflections and local changes in the magnetic properties of the counter surface, the signal of the proximity sensor may oscillate during a single stroke significantly. In order to compensate such 
local effects, the average value of $\delta h$ per stroke is used for indicating the evolution of the wear displacement. This per stroke averaging can also be seen as a filtering of the measured signal.

In comparison to other systems that record the wear displacement on the loading arm, the advantage of mounting the proximity sensor close to the pin sample is the minimization of possible errors from deformation and thermal expansion of the parts between the pin sample and the displacement sensor. Moreover, this setup utilizes directly the counter surface as the reference surface. In this way, many deviations of the involved components with respect to the fixed world reference are compensated mutually.

The impact of ambient temperature changes on the monitored gap could be estimated in the implemented test-rig by testing a sliding material under grease lubrication and wear rate much lower than for friction material in holding brakes. During a testing period of 55 hours, long-term fluctuations of the measured gap in the order of $4 \mathrm{\mu m}$ could be observed. Therefore, a reliable wear rate estimation requires sufficient testing distance for the total wear displacement to be at least one order of magnitude higher.

The choice of Eddy-current proximity sensors instead of capacitive sensors was based on their lower sensitivity to contaminants on the target surface. Their main disadvantage is that they normally have to be pre-calibrated by the manufacturer for a specific counter surface material, which additional has to be conductive. For testing different friction materials running against a steel surface, which is the typical case in holding brake application, these limitations are of less importance.

\subsection{Specimen preparation and installation}

Pin samples are normally machined by turning either a bar or a plate of the raw material. Unless otherwise required, the pin axis should correspond to the axis of the raw material bar or it should be perpendicular to the mean plane in case of raw material plates.

The surface of the insert plate (8) can be either ground or also polished if required. Polished surfaces are appropriate for fundamental tribological testing, whereas simply ground surfaces are relevant for testing with respect to a specific real life application with similar roughness characteristics, [5].

Both the pin sample and the corresponding plate have to be cleaned before testing. Cleaning with ethyl alcohol and consequently drying can be repeated one or more times. Cleaning in ultrasound bath and drying in an oven is recommended by the ASTM standards [4] and [5]. For each test, an unused track on plate (8) has to be used. Reusing the same track for tests with different pin materials should be avoided, because, in most cases, the presence of an already formed transfer film is expected to have a non-negligible impact on the new test.

In order to ensure a correct operation of the test-rig a few alignments are required before each test. As a first step, a digital level is used in order to check that the pivot points (A) and (B) of Fig. 1 are aligned on the same vertical line. If not, the distance $\mathrm{CD}$ is adjusted by modifying the length of the connectors of load-cell (4).

In a second step, in order to avoid self energizing and de-energizing of the friction force, it is checked that the free surface of pin (1a) and the center of pivot (A) lie in the same horizontal plane, when the loading arm (2) is horizontal. If not, screw (10) is adjusted until this condition is fulfilled. Next, it is checked that when the pin is loaded 
against plate (8), the loading arm is horizontal and the pressure on the pin surface is uniform. For this purpose, pressure sensitive paper is put between pin (1) and plate (8) and the height of the sub-frame (6) is adjusted iteratively until a uniform imprint of the pin on the pressure sensitive paper is achieved, see Fig. 3 .

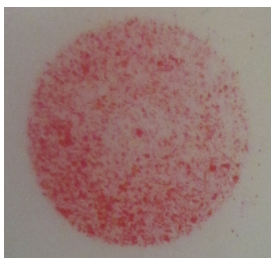

Figure 3: Pin imprint on pressure sensitive paper, after alignment

\subsection{Normal load calibration}

The normal load applied on the pin could theoretically be calculated based on the weight and center of gravity of arm (2) along with the weight and position of mass (3). However, in order to avoid the complex calculation of the arm center of gravity and account for deviations in the geometry and density of the involved parts, it is more practical to use a compressive load-cell placed below the pin and move the dead weight until the recorded normal force corresponds to a predetermined value. In this way, one can mark several positions of the dead weight on the arm, which provide the required pressure levels on the pin surface.

\subsection{Comparative advantages and disadvantages}

Depending on the materials to be tested as well as the load, speed and environmental conditions, different experimental setups may be preferred for friction and wear testing. However, the same fundamental difficulties have to be overcome in almost every case. For measuring the friction force it is necessary to incorporate a force transducer in the structure supporting one of the contacting bodies, parallel to the sliding direction. This force transducer has to be incorporated in such a manner that it will not compromise the stiffness of the support and no significant tilting between the contacting bodies will occur. For monitoring the wear displacement it is essential to minimize the impact of thermal expansion and deflection of other components on the measurement. Some of the advantages and disadvantages of the proposed test-rig concept with respect to the above mentioned aspects are summarized below:

+ Inexpensive longitudinal force transducers can be utilized.

+ The friction force in the contact does not contribute to tilting of the pin specimen.

+ Deviations with respect to the fixed world reference are partially compensated in the wear displacement measurement because the latter is based on the relative position of the pin and the counter surface.

+ The proposed test-rig concept is suitable for an implementation with multiple pins tested in parallel. 
- Good alignment of pivot points (A) and (B) is essential for the accuracy of the friction force measurement.

- Friction losses in pivots (A) and (B) affect the applied normal force and the measured friction force respectively.

- Wear monitoring though Eddy-current proximity sensors is possible only for conductive counter surface materials.

- Eddy-current proximity sensors need to be pre-calibrated for a specific counter surface materials.

Many of these characteristics also apply to reciprocating test configurations described in references [2], 5] and [8. However, the proposed concept is believed to combine many of the positive characteristics, while its negative aspects are normally of little or no relevance for the study of friction materials for holding brake applications.

\section{Results and discussion}

In this section representative testing results from a quadruplet pin-on-plate test-rig are presented. In each test, four pins made of the same material were tested in parallel. Since the environmental conditions are common for each group of pins, the observed differences among them can be attributed either to random differences in the material composition and the counter surfaces or to inaccuracies in the test-rig itself and the calibration of its sensors.

The first example shows detailed testing results for glass and aramid fiber reinforced polyamide 6.6 (PA66) as the pin material. In the second example, more synoptic results of friction force and wear evolution for pins made of polyethylene-terephthalate (PET) are presented. For the latter case, the final wear height measured through the Eddy-current proximity sensors is compared with two conventional methods, pin weighing and pin height measurement using a coordinate measuring machine (CMM). The pin dimensions are those given in section 2.2

In all cases, the counter part corresponding to part (8) of Fig. 1 is made of alloy steel $42 \mathrm{CrMo} 4$ in unhardened condition and its functional surface is ground in the direction parallel to the sliding. The arithmetic average roughness Ra, was measured on each plate at six different positions in direction perpendicular to the sliding. Despite the fact, that all eight plates used in both tests presented below were ground in the same pass of the grinding machine, the mean Ra value per plate varied from 0.48 to $0.76 \mu \mathrm{m}$.

The stroke length for both tests presented below was $0.668 \mathrm{~m}$. When referring to stroke counting, one stroke is meant as a complete forward and reverse movement corresponding to a cumulative sliding distance of $1.336 \mathrm{~m}$.

\subsection{Testing of reinforced PA66}

Reinforced PA66 pins were tested under nominal pressures of 6, 9 and $12 \mathrm{MPa}$ and a sliding velocity of $20 \mathrm{~mm} / \mathrm{s}$. The results presented below refer to the highest load case of $12 \mathrm{MPa}$. Figures 4 and 5 show the evolution of the measured friction force and wear displacement respectively, in two individual strokes for the first of the four pins. 


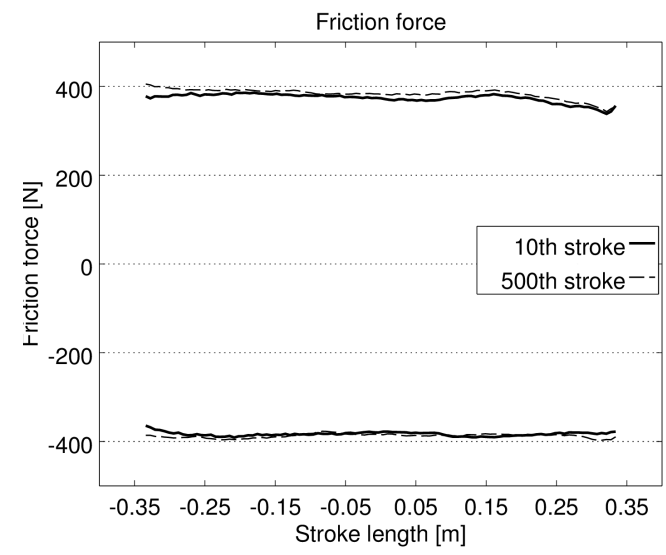

Figure 4: Measured friction force in the 10th and the 500th stroke for the first pin of reinforced PA66 at $12 \mathrm{MPa}$

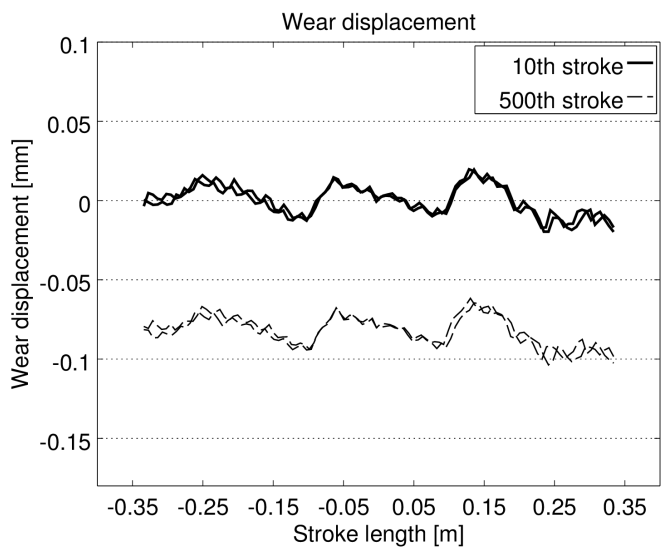

Figure 5: Measured wear displacement in the 10th and the 500th stroke for the first pin of reinforced PA66 at $12 \mathrm{MPa}$

In Fig. 4 one can recognize the forward and reversing part of the stroke based on the sign of the measured force. There are no significant differences between the 10th and the 500 th stroke. This indicates that the contact between the pin and the plate at the 10th stroke was already in steady state. This is because of the testing at 6 and $9 \mathrm{MPa}$ that occurred prior to the here presented test at $12 \mathrm{MPa}$.

Fig. 5 illustrates fluctuations in the measured wear displacement that may be observed within each individual stroke. In section 2.2, such discrepancies were attributed to geometrical deviations, elastic deflections and local variation of magnetic properties. The results presented here show that the fluctuation pattern is very reproducible even after $1 \mathrm{~km}$ of testing. Moreover, the differences between forward and reversing directions are very small. Based on these observations, it is expected that, despite the important variation of the apparent wear displacement within each stroke, the offset between per 
stroke averages can represent the wear displacement adequately. It should be noted, that by considering the 10th stroke as a reference, the average wear displacement for this stroke in Fig. 5 is by definition equal to zero.

Figures 6 and 7 show the overall evolution of the friction force and the wear displacement respectively, for all four pins during the $1 \mathrm{~km}$ long testing at $12 \mathrm{MPa}$. Each single point in these curves is an average value per stroke. This means that each curve of Fig. 4 or Fig. 5 is represented by its average as a single point in Fig. 6 or Fig. 7 respectively. Especially for negative friction forces, their absolute value is considered.

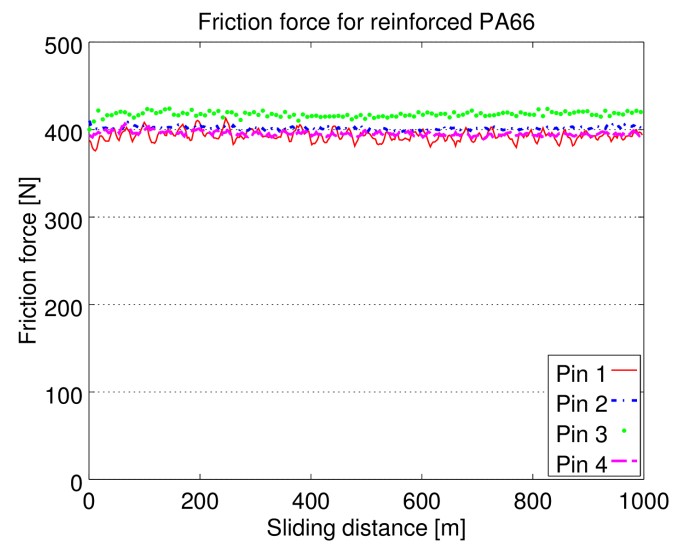

Figure 6: Evolution of friction force for reinforced PA66 at $12 \mathrm{MPa}$

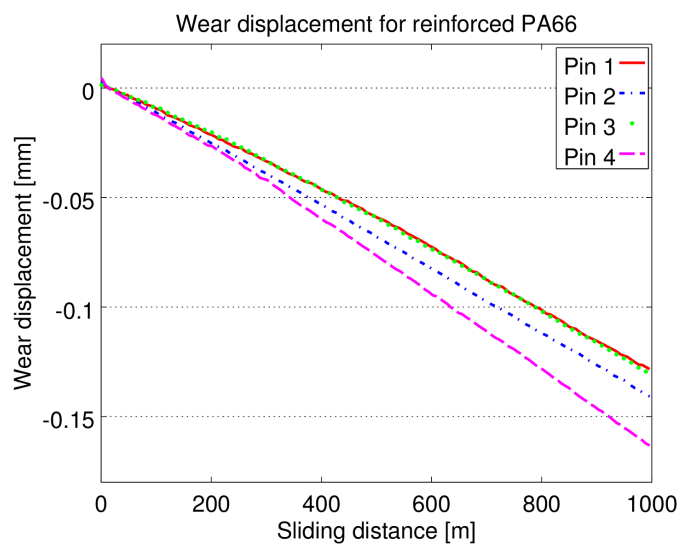

Figure 7: Evolution of wear displacement for reinforced PA66 at $12 \mathrm{MPa}$

Table 1 summarizes wear rate and coefficient of friction values extracted from Figures 7 and 6 . The wear rate values presented here correspond to the slope of a line fitted to the wear displacement curves within the last $500 \mathrm{~m}$ of testing. The calculated coefficients of friction are based on the average friction force in the last $500 \mathrm{~m}$ of testing, divided by the prescribed normal force of $943 \mathrm{~N}$ that corresponds to a nominal pressure 
of $12 \mathrm{MPa}$. The coefficient of variance for the four presented wear rate and coefficient of friction values is 10.6 and $2.8 \%$ respectively. It should be noted that the here tested variant of fiber reinforced PA66 was also included in the pin-on-disc testing presented in [3]. Despite the different surface finishing of the steel plates used in the here presented tests and the steel disc used in $\underline{3}$, the reported friction coefficient of 0.4 at $12 \mathrm{MPa}$ from reference [3, is very close to the average friction coefficient of 0.43 reported in Table 1 .

\begin{tabular}{lcc} 
& $\begin{array}{c}\text { Wear rate } \\
{[\mu \mathrm{m} / \mathrm{km}]}\end{array}$ & $\begin{array}{c}\text { Coefficient } \\
\text { of friction }\end{array}$ \\
\hline Pin 1 & 139 & 0.42 \\
Pin 2 & 146 & 0.43 \\
Pin 3 & 141 & 0.44 \\
Pin 4 & 173 & 0.42 \\
\hline Average & 150 & 0.43
\end{tabular}

Table 1: Wear rate and coefficient of friction for reinforced PA66 at $12 \mathrm{MPa}$

\subsection{Testing of PET}

Figures 8 and 9 show the evolution of the friction force and the wear displacement respectively, during a $5.3 \mathrm{~km}$ long test of PET pins under a nominal pressure of $6 \mathrm{MPa}$ and sliding velocity of $20 \mathrm{~mm} / \mathrm{s}$.

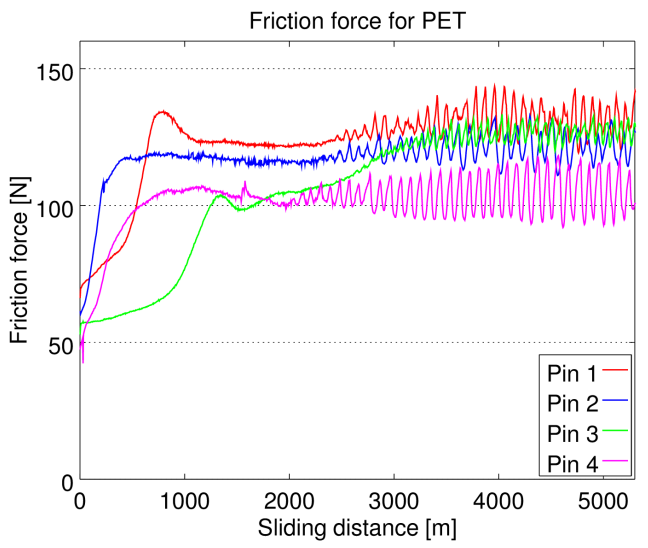

Figure 8: Evolution of friction force for PET at $6 \mathrm{MPa}$ 


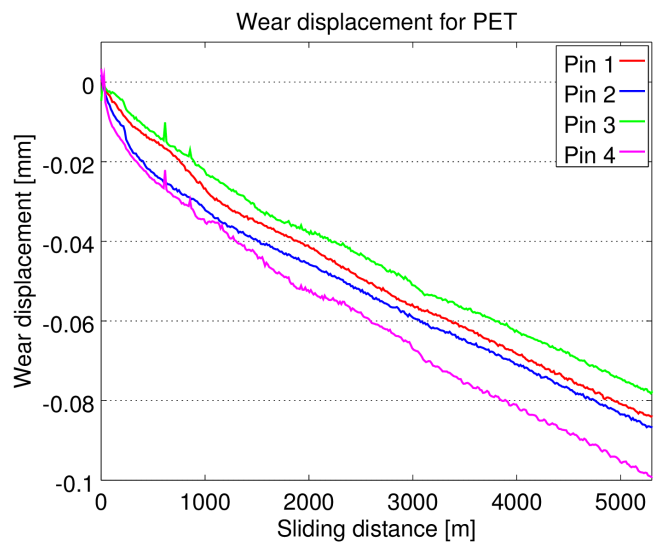

Figure 9: Evolution of wear displacement for PET at $6 \mathrm{MPa}$

Regarding the friction force graph in Fig. 8 , two phenomena can be observed. During the first $1.5 \mathrm{~km}$ the contact between pin and moving plate runs in and the friction force is approximately doubled with respect to its initial level. The running-in distance varies among the four pins from 0.5 to $1.5 \mathrm{~km}$. The second phenomenon is observed in the last $3 \mathrm{~km}$. It is a periodic build up and decrease of the friction force. The period of this variation is about 75 strokes corresponding to approximately $100 \mathrm{~m}$ of sliding distance which is covered in circa 1.5 hours. It is assumed that the cause of such oscillation is an unstable transfer layer thickness on the steel surface. However, further investigation is required in order to prove this assumption. Both the period and the amplitude of the friction force oscillation vary among the four pins.

Table 2 summarizes the average wear rate and coefficient of friction values estimated in the last $500 \mathrm{~m}$ of the graphs in Figures 9 and 8 . The coefficient of variance for the four presented wear rate and coefficient of friction values is 8.8 and $9.6 \%$ respectively.

\begin{tabular}{lcc} 
& $\begin{array}{c}\text { Wear rate } \\
{[\mu \mathrm{m} / \mathrm{km}]}\end{array}$ & $\begin{array}{c}\text { Coefficient } \\
\text { of friction }\end{array}$ \\
\hline Pin 1 & 11.7 & 0.28 \\
Pin 2 & 12.3 & 0.26 \\
Pin 3 & 11.9 & 0.27 \\
Pin 4 & 14.1 & 0.22 \\
\hline Average & 12.5 & 0.26
\end{tabular}

Table 2: Wear rate and coefficient of friction for PET at $6 \mathrm{MPa}$

Table 3 compares three different methods for estimating the total wear height. The wear values according to the proximity sensors method correspond to the final point of the curves in Fig. 9 .

Regarding the weighing method, the four pins, including the pin holder (1b) of Fig. 2 were weighed before and after the test. The pin holder (1b) was made of aluminum in order to reduce the total weight of the compound and increase the accuracy of weighing. Before each weighing the pins were repeatedly cleaned with isopropyl alcohol and dried 
with pressurized air. A weighing device with a resolution of $1 \mathrm{mg}$ was used and the observed weight differences were converted to equivalent heights under consideration of the pin diameter of $10 \mathrm{~mm}$ and a density of $1.38 \mathrm{mg} / \mathrm{mm}^{3}$ for the PET material.

\begin{tabular}{cccc} 
& \multicolumn{2}{c}{ Total wear height $[\mu \mathrm{m}]$} & \\
& Proximity sensors & Weighing & CMM \\
\hline Pin 1 & 85 & 83 & 92 \\
Pin 2 & 88 & 88 & 91 \\
Pin 3 & 79 & 74 & 79 \\
Pin 4 & 100 & 92 & 95
\end{tabular}

Table 3: Total wear height for PET at $6 \mathrm{MPa}$

Regarding the CMM pin height measurement, the surface of the pin holder (1b) was used as a reference by calculating the average plane of points probed at a radius of $20 \mathrm{~mm}$, see Figures 10 and 2. In order to specify the height of the protruding part of pin (1a) with respect to the pin holder (1b), nine points on the pin surface, illustrated in Fig. 10, were probed. The pin height was evaluated as the average z-coordinate of these nine probed points. Carrying out the height measurement for each pin before and after the test gave the possibility to calculate the wear height as the corresponding heights difference.

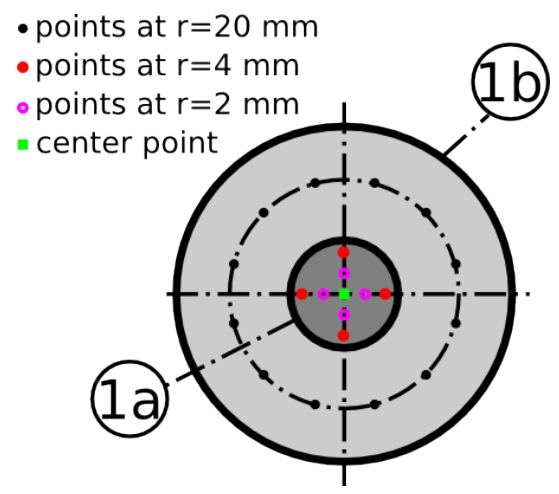

Figure 10: Probing points for the pin height measurement with CMM.

The CMM method is considered as the most accurate one and consequently the third column of Table 3 will be used as a reference for the other two methods. A paired Student's t-test between columns one and three yields a t-value of -0.49 , whereas a comparison between columns two and three gives a t-value of -3.54. For a significance level of $10 \%$ and the given number of samples the confidence interval for the t-value is between -2.35 and 2.35. This means that no systematic difference between the Eddycurrent sensor and the CMM method could be detected. On the other side, the differences between the weighing method and the CMM method are statistically significant. The weighing method seems to underestimate the reduction of the pin height. This can be explained by the fact that the measurand in this case is the mass, whereas the other two methods both involve the pin height directly. The assumed relation between mass 
and height reduction relies on a previously determined material density and neglects any possible plastic deformation of the pin sample.

Another interesting observation is that even for a very homogeneous material like PET, there seem to be important deviations between different pin samples. This fact underlines the importance of running multiple tests under the same conditions in parallel, in order to obtain a minimal statistical sample to draw conclusions from.

Apart from verifying the in situ wear displacement monitoring results, the CMM pin height measurements can also be utilized for examining the form of the pin surfaces after testing. For each of the four tested PET pins, Fig. 11] shows the surface profile along the sliding direction. The five points defining each curve correspond to the five probe points on the horizontal symmetry line of Fig. 10 All four pin surfaces exhibit a convex form which is due to tilting of the pin because of elastic deformations of the test-rig. However, the deviations from the flat form are limited to less than $2 \mu \mathrm{m}$ on a diameter of $8 \mathrm{~mm}$, which is indicative for the very stiff implementation of the presented test-rig.

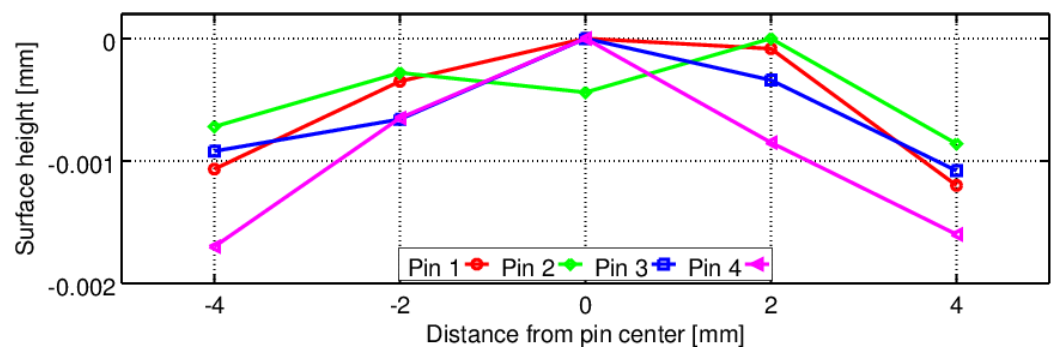

Figure 11: Profiles of the PET pin surfaces along the sliding direction.

\section{Conclusions}

In this paper a novel pin-on-plate test-rig concept was presented along with corresponding examples of measured friction and wear. The novelty of the design consists in the way that the friction force is transferred to a load-cell, while at the same time self energizing and de-energizing of the friction force between the pin and the plate is avoided.

Moreover, a successful implementation of in situ wear displacement monitoring based on Eddy-current proximity sensors was reported. Details about the mounting of such sensors and the interpretation of the measured signal were presented. The obtained final wear heights were compared to classical methods like weighing and CMM dimensional measurements on the pins. A good correlation between the results from the proximity sensors system and the CMM pin height measurements could be found, whereas the weighing method seemed to underestimate the reduction of the pin height.

Apart from demonstrating the operation of the presented test-rig, the included testing results are also relevant for holding brake applications, providing reference values for the coefficient of friction and wear rate of two thermoplastic material types running against a ground steel surface. 


\section{References}

[1] J. Byett, C. Allen, Dry sliding wear behaviour of polyamide 66 and polycarbonate composites, Tribology International 25 (1992) 237-246.

[2] R. Bayer, Mechanical Wear Fundamentals and Testing, Revised and Expanded, Dekker Mechanical Engineering, Taylor \& Francis, 2004.

[3] K. Poulios, G. Svendsen, J. Hiller, P. Klit, Coefficient of friction measurements for thermoplastics and fibre composites under low sliding velocity and high pressure, Tribology Letters (2013).

[4] ASTM International, G99-05 standard test method for wear testing with a pin-on-disk apparatus, 2010.

[5] ASTM International, G133-05 standard test method for linearly reciprocating ball-on-flat sliding wear, 2010.

[6] M. Neale, M. Gee, Guide to wear problems and testing for industry, William Andrew Pub, 2001.

[7] J. Fraden, Handbook of Modern Sensors. ; Physics, Designs, and Applications, Springer, 2010.

[8] G. Stachowiak, A. Batchelor, Experimental Methods in Tribology, Tribology and Interface Engineering, Elsevier Science, 2004. 